\title{
Modeling Clustered-Hole Nozzle Sprays Using Advanced Liquid-Gas Phase Coupling Method
}

\author{
Sungwook Park, Daesik Kim, Hyungu Roh, and Mun Soo Chon
}

\begin{abstract}
This paper describes updated method to modeling clustered-hole nozzle sprays. The clustered-hole nozzle was suggested to improve atomization performances without sacrificing spray tip penetration. It has been well-known that the spray tip penetration is significantly important because it plays an important role in diesel engine performances. Thus, clustered-hole nozzle layout exhibited improved engine performances.

In the present study, advanced spray models have been suggested for modeling clustered-hole nozzle based on the gasjet model. Results show that the predicted results with the present model exhibit minimal discrepancies compared with experimental results.
\end{abstract}

Keywords - Clustered-hole nozzle, spray model, KIVA code

\section{INTRODUCTION}

Diesel engines show better fuel economy compared to gasoline engines mainly due to higher compression ratio. However, in diesel engines, fuel should be evaporated and mixed with air within limited time. Thus, fuel spray atomization is significantly important $[1,2]$.

Despite CFD (computational fluid dynamics) is able to use effectively in developing internal combustion engine, it exhibit high grid size dependency due to liquid-gas momentum exchange process. Therefore, grid-size independent spray models are required for using CFD in developing clustered-hole nozzle.

This paper describes application process of updated spray models composed of the gas-jet model, radius-of-influence collision and coalescence, mean collision time and improved interpolation method. The updated spray models were used for modeling non-evaporating clustered-hole nozzle sprays.

\section{SPRAY MODELS}

\section{A. Gasjet model}

The gas-jet model calculates the ambient gas velocity as a function of distance from the nozzle exit based on the gas-jet

Manuscript received December. 9, 2015.

S. Park is with School of Mechanical Engineering, Hanyang University, Seoul, Korea.

D. Kim is with School of Mechanical and Automotive Engineering, Gangneung-Wonju National University, Wonju, Korea

H. Roh is with Department of Mechanical \& Automotive Engineering, Induk University, Seoul, Korea

M. S. Chon is with Department of Energy System Engineering, Korea National University of Transportation, Chunju, Korea theory suggested by Abraham [3] and Schlichting [4], and does not use the CFD prediction. The ambient gas velocity along the spray axis, , is calculated from

$$
V_{\text {gasjet }}=\frac{3 U_{i n j} d_{n o z} \sqrt{\frac{\rho_{l}}{\rho_{g}}}}{K_{\text {entr }} Z}\left(\frac{1}{\left(1+\frac{12 R^{2}}{K_{\text {entr }}^{2} Z^{2}}\right)^{2}}\right)
$$

\section{B. Mean collision time model}

In the present paper, the mean time for collision ( ) is calculated for each cell from ref. [5]. The advantage of this model is that it can overcome this time-step dependency.

$$
d t_{c o l}=\frac{0.4}{u_{\max , \text { cell }}}\left(\frac{R O I}{N_{c v}^{1 / 3}}\right)
$$

where $u_{\text {max }, \text { cell }}$ is the maximum velocity with in the cell and $N_{c v}$ is the number of parcels within ROI (radius-of-influence). If computational time step is greater than mean collision time, time step is reduced to mean collision time. On the other hand, if time step is less than mean collision time, collisions are carried out only when an accumulated time is reached mean collision time.

C. Clustered-hole nozzle layouts

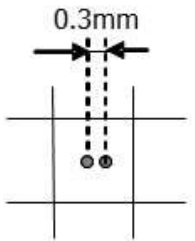

(a) Layout 1

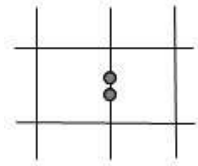

(c) Layout 3

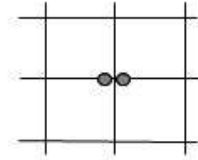

(b) Layout 2

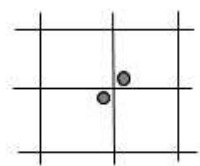

(d) Layout 4
Fig. 1: Layouts of clustered-hole nozzle

Figure 1 shows layout of cluster-hole nozzle layout. In the present study, four kinds of nozzle layouts were considered in order to investigate effects of nozzle hole layouts on spray characteristics. 


\section{RESULTS AND DISCUSSIONS}

Figures 2 and 3 show hole-location and grid-size on spray tip penetration. In this figure, it can be seen that spray tip penetration is highly dependent on both hole-location and grid-size dependency. Previous studies [5] showed that the hole-location and grid-size dependency are cause by the inaccurate calculation of liquid-gas momentum exchange process. For conventional KIVA models, the spray penetrations become shorter with large computational cell sizes because the smaller cell size leads to higher gas velocities along the spray axis during the momentum exchange process, and this causes increased penetration of the spray.

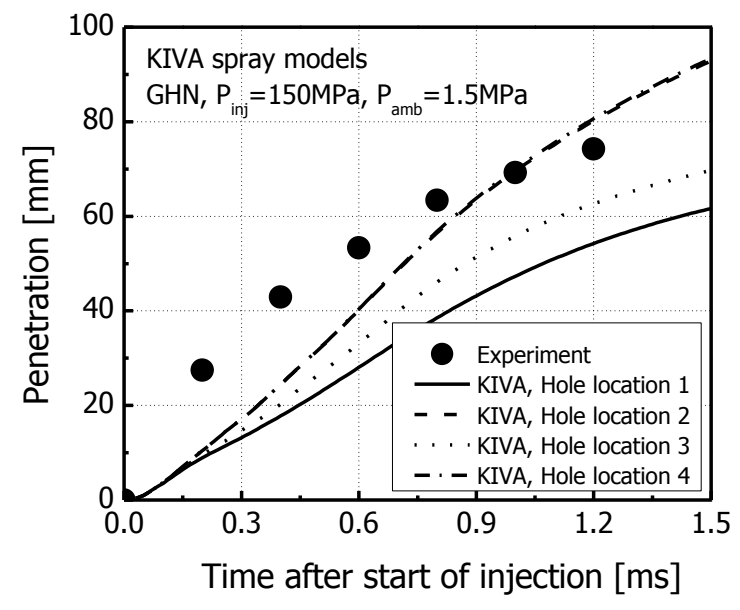

Fig. 2: Hole-location dependency on spray tip penetration for various hole locations

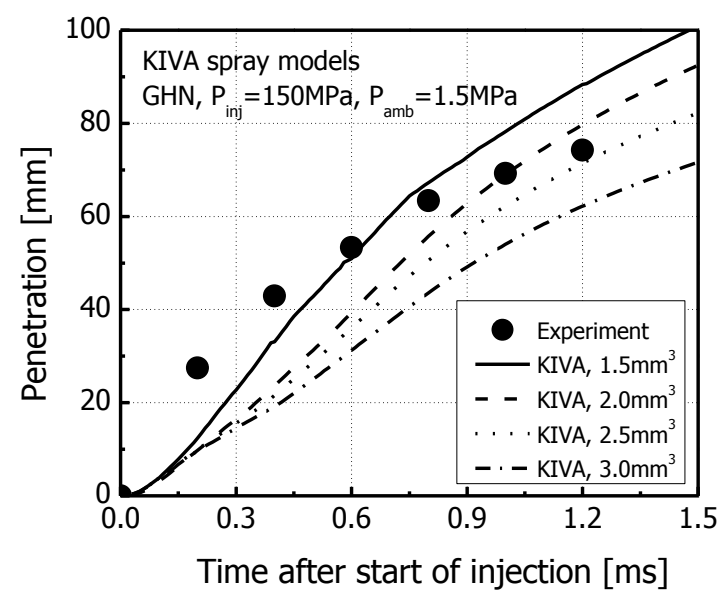

Fig. 3: Grid-size dependency of conventional KIVA models

In Fig. 4, it can be seen that the grid-size dependency is almost eliminated for updated spray models. Thus, it is believed that grid-size dependency of spray penetration in simulating clustered-hole nozzle sprays was reduced significantly by introducing the new spray models.

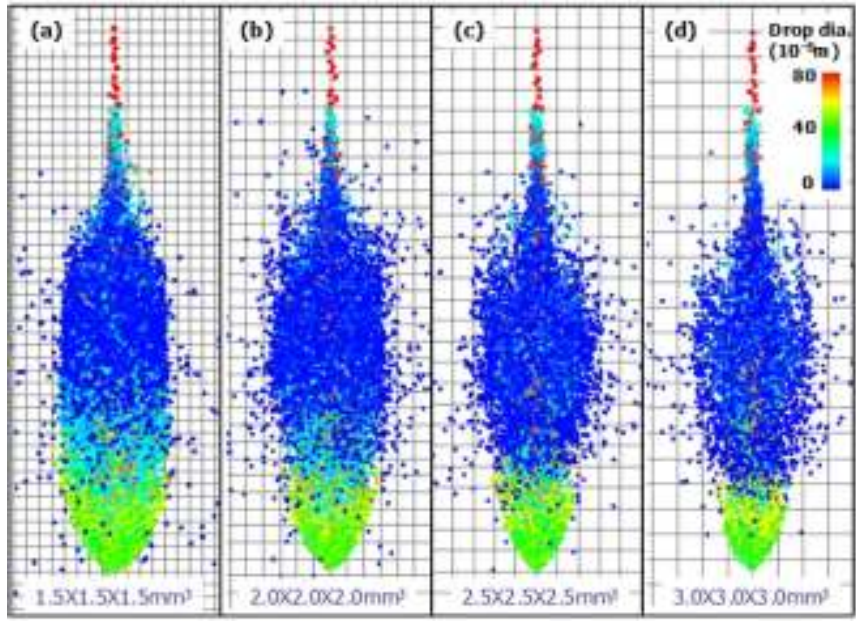

Fig. 4: Spray development process for updated spray models

\section{CONCLUSION}

In original KIVA spray models, significant dependencies of grid-sizes and hole-locations on clustered-hole nozzle sprays were found with standard spray models. The dependencies mainly arise from inaccuracies in calculating the momentum exchange and droplet-droplet collision. Grid-size dependency of spray penetration in simulating clustered-hole nozzle sprays was reduced significantly by introducing the new spray models of the present study.

\section{REFERENCES}

[1] Park, S.W., Internal Structure and Atomization Characteristics of High-Pressure Injection Diesel Sprays, Ph.D. Thesis, Department of Mechanical Engineering, Hanyang University, 2005.

[2] Wickman, D.D., Tanin, K.V., Senecal, P.K., Reitz, R.D., Gebert, K., Barkhimer, R. L., Beck, N. J., Methods and Results from the Development of a 2600 bar Diesel Fuel Injection System, SAE paper 2000-01-0947, 2000.

[3] Abraham, J., Entrainment Characteristics of Transient Jets, Numerical Heat Transfer, Part A, Vol. 30, pp. 347-364, 1996.

[4] Schlichting, H., Boundary Layer Theory, McGraw Hill, NY, USA., 1976.

[5] Munnannur, A., Droplet Collision Modeling in Multi-dimensional Engine Spray Computations, Ph.D. thesis, Department of Mechanical Engineering, University of Wisconsin-Madison, 2007 